\title{
Harnessing Data Science Through Healthcare IT Interoperability
}

\section{Sheryl L. Taylor}

National Institute of Standards and Technology, Gaithersburg, Maryland, United States

Objective

To provide tools to generate national and local syndromic surveillance electronic messaging specifications and to test implementations in which the set of requirements have been implemented in order to confirm or refute the conformance to those requirements, thereby promoting healthcare information technology (HIT) interoperability in the public health sector.

\section{Introduction}

The ability to harness data science for use in improving population health and public health surveillance begins with the application of interoperability standards to electronic messaging for data exchange between HIT used by public health authorities (PHAs) and the providers who submit patient data to them. When electronic transmissions between these entities are not based on interoperability standards, the patient data that are exchanged may be incomplete, inaccurate, invalid, and/or untimely. As a result, local PHAs and the Centers for Disease Control and Prevention (CDC) may be unable to fulfill their goals of monitoring public health trends and improving population health.

\section{Methods}

As part of the effort to meet the need for the application of interoperability standards to electronic messaging for data exchange between HIT modules that submit and collect syndromic surveillance data for public health, the National Institute of Standards and Technology (NIST), in collaboration with the CDC and the International Society for Disease Surveillance (ISDS), developed and maintains a set of validation tools. These tools are focused on standardized syndromic surveillance messaging and are used for HIT certification testing by the Office of the National Coordinator (ONC) and for on-boarding by various public health jurisdictions in the US. In addition, ISDS informatics personnel are using the NIST Implementation Guide Authoring and Management Tool (IGAMT) for creating the first HL7-ballotted version of a guide for syndromic messaging, the HL7 2.5.1 Implementation Guide for Syndromic Surveillance Release 1. This guide is a messaging specification that defines how disparate healthcare applications are to codify and transmit administrative and clinical data for public health surveillance and response. IGAMT is part of an integrated platform that also includes the NIST Test Case Authoring and Management Tool (TCAMT), a Testing Infrastructure and Framework, and the NIST General Validation Tool (GVT). This Web-based platform enables domain experts, such as the ISDS informatics experts, to control the automatic process for generating computable standards and associated testing tools.

\section{Results}

Developed through collaboration between NIST, the CDC, and ISDS, the 2015 Edition Syndromic Surveillance Test Suite has been used in the ONC HIT Certification Program for validating over one hundred HIT modules against the syndromic messaging guide developed by the CDC and the Public Health Information Network, the PHIN Guide for Syndromic Surveillance Messaging Release 2.0 and the associated Erratum. During the collaborative process, NIST contributed expertise based on many years of co-authoring and using HIT interoperability specifications, and the CDC and ISDS contributed expertise pertaining to the syndromic surveillance domain. Outcomes of this process included increased awareness by all involved parties regarding the challenges of writing computable standards and the challenges associated with testing HIT under constrained circumstances, such as with the ONC HIT Certification Program. The recognition of the need for well-defined standards, as well as testing using real-world scenarios and clinical data, led to the development of IGAMT and TCAMT for automating the production of these artifacts; and with these tools came the ability to automate generation of testing resources, such as syndromic surveillance validation tools that are customized to national-level specifications as well as to state/local-level specifications for use in on-boarding procedures. As of early 2017, states with jurisdictions requiring providers to validate the ability of their HIT modules to generate syndromic messages using the NIST national-level Syndromic Surveillance Test Suite in their on-boarding process included Arkansas, Florida, Indiana, Kansas, Maryland, South Carolina, and Washington. Now that a national-level HL7- balloted syndromic surveillance implementation guide has been generated using IGAMT, representatives of several additional PHAs have expressed interest in using the components of 
the NIST Integrated Platform for generating local-level specifications and testing tools. State and local jurisdictions often require certain data to be submitted in addition to the data required by the national-level specification. Local-level testing tools used during the on-boarding process would enable jurisdictions to validate syndromic messages created by submitters in order to confirm or refute the conformance to the local-level requirements.

\section{Conclusions}

Improving population health and public health surveillance by utilizing the power of data science requires the ubiquitous deployment of standards- based data exchange, that is, interoperability, between the numerous disparate HIT modules in use by providers and PHAs today. NIST has created a development platform that enables the domain experts at the CDC and ISDS to use automated tools to generate national- and local-level syndromic surveillance electronic messaging specifications and the associated testing tools that confirm or refute conformance to the requirements in these specifications. These tools promote interoperability as the foundation for harnessing data science for the benefit of the public and the public health entities that serve them.

\section{Acknowledgement}

This effort is accomplished by the National Institute of Standards and Technology in collaboration with the Centers for Disease Control and Prevention and the International Society for Disease Surveillance. 